\title{
Eosinophilie als Asthma-Risikofaktor
}

\author{
Gibt es einen Zusammenhang zwischen einer Eosinophilie im \\ peripheren Blut und dem Asthmarisiko bei Kindern? Dieser Frage \\ gingen Wissenschaftler aus Arizona im Rahmen einer retro- \\ spektiven Studie nach. Besonderes Augenmerk richteten sie auf die \\ Rolle atopischer Erkrankungen in der Asthma-Pathogenese.
}

n die Langzeit-Kohortenstudie wurden insgesamt 1.246 zunächst gesunde Säuglinge einbezogen, die bis zu ihrem 11. Lebensjahr mehrmals untersucht wurden. Die Untersuchungen schlossen mehrere Prick-Tests zur Ermittlung von Allergien ein. Bei einem positiven Test wurde das Kind als ,atopisch" eingestuft. Regelmäßige Befragungen der Eltern sollten eine eventuelle Asthmadiagnose des Kindes in Erfahrung bringen. Je nach eosinophilen Granulozyten im Blut, wurden die Kinder in vier verschiedene Gruppen eingeteilt: anhaltend niedrige ( $\leq 2 \%)$, intermittierend niedrige (1-2 von 3 Untersuchungen $\leq 2 \%$ ), intermittierend hohe (1-2 von 3 Untersuchungen $>5 \%)$ oder anhaltend hohe Eosinophilenzahl (> 5\%).

Je höher die Zahl der eosinophilen Granulozyten im Blut gemäß dieser Abstufung war, umso mehr Kinder der je- weiligen Gruppe erkrankten an chronischem Asthma: Die Prävalenz betrug 5,8\% bei Kindern mit anhaltend niedriger Eosinophilenzahl und steigerte sich bis zu 37,5\% in der Gruppe mit anhaltend hoher Eosinophilenzahl. Diese Zunahme trat sowohl bei Kindern ohne wie auch bei Kindern mit Atopie auf, bei letzteren mit einem höheren Anteil an chronischen Asthmaerkrankungen. Die Wissenschaftler folgern, dass Kinder mit atopischen Erkrankungen und einer persistierenden Eosinophilie möglicherweise das größte Risiko besitzen, an Asthma zu erkranken.

Kinder, deren Eltern an Asthma erkrankt waren, zeigten außerdem höhere Eosinophilenwerte als Kinder ohne entsprechende Familienanamnese ( $\mathrm{p}=$ 0,001). Die Erkrankungsrate für Asthma war bei den Kindern mit asthmakranken Eltern ebenfalls signifikant erhöht. Die Forschergruppe vermutet

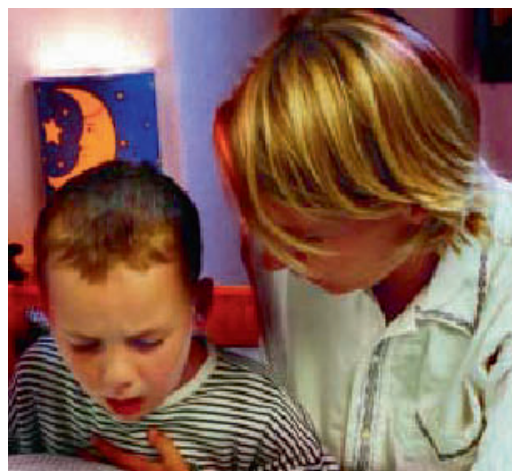

Atopie und Eosinophilie könnten unabhängige und zugleich vererbbare Asthma-Risikofaktoren sein.

aufgrund dieser Ergebnisse, dass der Eosinophilenstatus vererbbar sein könnte.

Fazit: Noch ist unklar, ob hohe Werte von eosinophilen Granulozyten lediglich eine Störung des Immunsystems darstellen oder direkt an der Pathogenese von Asthmaerkrankungen beteiligt sind. Die Eosinophilenzahl könnte als aussagekräftige Größe bei der Einschätzung des Asthmarisikos von Kindern fungieren.

$n z$

Karakoc F. et al. The association between persistent eosinophilia and asthma in childhood is independent of atopic status. Clin Exp Allergy 2002; 32: 51-6

\section{Etagenwechsel rechtzeitig verhindern}

\section{Erwachsene, die nicht an Asthma, jedoch an allergischer Rhinitis leiden, zeigen bei bronchialen Provokationstests mit Histamin und Methacholin eine erhöhte Obstruktion. Ist dies auch im Kindesalter bereits der Fall?}

P neumologen und Immunologen der Berliner Charité untersuchten 654 Kinder im Alter von sieben Jahren, die an der German Multicenter Allergy Study teilnahmen. Dabei dokumentierten sie die Provokationsdosis von inhaliertem Histamin, die zu einem Abfall der forcierten Expiration nach einer Sekunde $\left(\mathrm{FEV}_{1}\right)$ um 20\% führte. Sie verglichen diese Werte bei Kindern mit Asthma, mit allergischer
Rhinitis, mit asymptomatischer Sensibilisierung und ohne Hinweis auf Atopie.

Die ausgeprägteste Reaktion auf Histamin fand sich bei Kindern mit allergisch bedingtem Asthma, unabhängig davon, ob eine allergische Rhinitis bestand oder nicht. Interessant war auch, dass sich die Provokationsdosen bei Kindern mit allergischer Rhinitis, asymptomatischer Sensibilisierung oder ohne Atopie nicht signifikant voneinander unterschieden.

Fazit: Siebenjährige Kinder, die nicht an Asthma, sondern nur an allergischer Rhinitis leiden, zeigen im Gegensatz zu Erwachsenen noch keine erhöhte Reagibilität im bronchialen Provokationstest. Daher fordern die Autoren gerade für diese Kinder präventive Maßnahmen wie entsprechende Medikation oder Hyposensibilisierung, um rechtzeitig einen „Etagenwechsel“ zu verhindern

af

Nickel R et al. Comparison of bronchial responsiveness to histamine in asthma, allergic rhinitis and allergic sensitization at the age of 7 years. Clin Exp Allergy 2002; 32: 1274-77 